УДК 517.968.7

$$
\begin{aligned}
& \text { РЕШЕНИЕ ОАНОГО ГИПЕРСИНГУАЯРНОГО } \\
& \text { ИНТЕГРО-АИФФЕРЕНЦИААЬНОГО УРАВНЕНИЯ, } \\
& \text { ЗАААННОГО С ПОМОЩЬЮ ОПРЕАЕАИТЕАЕЙ }
\end{aligned}
$$

\author{
А. П. Шилин ${ }^{1)}$ \\ ${ }^{1)}$ Белорусский государственный университет, пр. Независимости, 4, 220030, г. Минск, Беларусь
}

\begin{abstract}
Приводится точное аналитическое решение гиперсингулярного интегро-дифференциального уравнения произвольного порядка на замкнутой кривой, расположенной в комплексной плоскости. Характерная особенность уравнения состоит в том, что оно записано с помощью определителей. С точки зрения традиционной классификации уравнений его следует отнести к линейным уравнениям с переменными коэффициентами специального вида. Применяется метод аналитического продолжения. Уравнение сводится к краевой задаче линейного сопряжения для аналитических функций с некоторыми дополнительными условиями. В случае разрешимости этой задачи требуется решить еще два линейных дифференциальных уравнения в классе аналитических функций. Указываются в явном виде условия разрешимости, при выполнении которых решение также может быть записано явно. Рассматривается пример.
\end{abstract}

Ключевые слова: интегро-дифференциальные уравнения; гиперсингулярные интегралы; обобщенные формулы Сохоцкого; дифференциальные уравнения; краевая задача Римана.

\title{
SOLUTION OF ONE HYPERSINGULAR INTEGRO-DIFFERENTIAL EQUATION DEFINED BY DETERMINANTS
}

\author{
A. P. SHILIN \\ ${ }^{\mathrm{a}}$ Belarusian State University, 4 Niezaliežnasci Avenue, Minsk 220030, Belarus
}

The paper provides an exact analytical solution to a hypersingular integro-differential equation of arbitrary order. The equation is defined on a closed curve in the complex plane. A characteristic feature of the equation is that if is written using determinants. From the view of the traditional classification of the equations, it should be classified as linear equations with variable coefficients of a special form. The method of analytical continuation id applied. The equation is reduced to a boundary value problem of linear conjugation for analytic functions with some additional conditions. If this problem is solvable, if is required to solve two more linear differential equations in the class of analytic functions. The conditions of solvability are indicated explicitly. When these conditions are met, the solution can also be written explicitly. An example is given.

Keywords: integro-differential equations; hypersingular integrals; generalised Sokhotsky formulas; differential equations; Riemann boundary problem.

\section{Образ ц цитирования:}

Шилин АП. Решение одного гиперсингулярного интегродифференциального уравнения, заданного с помощью определителей. Журнал Белорусского государственного университета. Математика. Информатика. 2021;2:17-28. https://doi.org/10.33581/2520-6508-2021-2-17-28

\section{For citation:}

Shilin AP. Solution of one hypersingular integro-differential equation defined by determinants. Journal of the Belarusian State University. Mathematics and Informatics. 2021;2:17-28. Russian.

https://doi.org/10.33581/2520-6508-2021-2-17-28

\footnotetext{
A в т о p:

Андрей Петрович Шилин - кандидат физико-математических наук, доцент; доцент кафедры высшей математики и математической физики физического факультета.
}

Author:

Andrei P. Shilin, PhD (physics and mathematics), docent; associate professor at the department of higher mathematics and mathematical physics, faculty of physics.

a.p.shilin@gmail.com 


\section{Введение}

В гиперсингулярных интегральных уравнениях (ГИУ) интегралы понимаются в смысле конечной части по Адамару. Основными методами решения таких уравнений являются численные методы, представление о которых можно получить, например, в статье [1]. Аналитические методы решения разработаны мало. Достаточно подробный обзор современного состояния методов решения ГИУ дан в работе [2], где, в частности, сказано: «В настоящее время ГИУ находят широкое применение при моделировании задач аэродинамики, электродинамики, микроэлектроники, геофизики, атомной и ядерной физики и ряда других областей естествознания и техники» [2, с. 245].

Точное аналитическое решение гиперсингулярного интегро-дифференциального уравнения впервые, по-видимому, приведено в статье [3] - это решение линейного уравнения на замкнутой кривой в комплексной плоскости в случае постоянных коэффициентов. В настоящей работе, как и в публикациях [4-6], аналогичное уравнение рассматривается для таких случаев переменных коэффициентов, когда возможность точного аналитического решения сохраняется.

\section{Постановка задачи}

Обозначим через $L$ простую гладкую замкнутую кривую на расширенной комплексной плоскости. Пусть $D_{ \pm}$- области, для которых кривая $L$ является границей, $0 \in D_{+}, \infty \in D_{-}$. Ориентируем кривую $L$ так, чтобы при движении по ней в положительном направлении область $D_{+}$оставалась слева.

Пусть $m, n \in \mathbb{N}$. Зададим $H$-непрерывные (т. е. удовлетворяющие условию Гёльдера) функции $a(t) \neq 0$, $b(t) \neq 0, f(t), m$ раз $H$-непрерывно дифференцируемые функции $p_{j}(t), j=\overline{1, m}$, и $n$ раз $H$-непрерывно дифференцируемые функции $q_{j}(t), j=\overline{1, n}, t \in L$. На кривой $L$ требуется найти $\max (m, n)$ раз $H$-непрерывно дифференцируемую функцию $\varphi(t)$, удовлетворяющую уравнению

$$
\begin{aligned}
& a(t)\left|\begin{array}{ccccc}
p_{1}(t) & p_{2}(t) & \ldots & p_{m}(t) & \varphi(t) \\
p_{1}^{\prime}(t) & p_{2}^{\prime}(t) & \ldots & p_{m}^{\prime}(t) & \varphi^{\prime}(t) \\
\ldots & \ldots & \ldots & \ldots & \ldots \\
p_{1}^{(m)}(t) & p_{2}^{(m)}(t) & \ldots & p_{m}^{(m)}(t) & \varphi^{(m)}(t)
\end{array}\right|+b(t)\left|\begin{array}{ccccc}
q_{1}(t) & q_{2}(t) & \ldots & q_{n}(t) & \varphi(t) \\
q_{1}^{\prime}(t) & q_{2}^{\prime}(t) & \ldots & q_{n}^{\prime}(t) & \varphi^{\prime}(t) \\
\ldots & \ldots & \ldots & \ldots & \ldots \\
q_{1}^{(n)}(t) & q_{2}^{(n)}(t) & \ldots & q_{n}^{(n)}(t) & \varphi^{(n)}(t)
\end{array}\right|+ \\
& +\frac{a(t)}{\pi i}\left|\begin{array}{ccccc}
p_{1}(t) & p_{2}(t) & \ldots & p_{m}(t) & 0 ! \int_{L} \frac{\varphi(\tau) d \tau}{\tau-t} \\
p_{1}^{\prime}(t) & p_{2}^{\prime}(t) & \ldots & p_{m}^{\prime}(t) & 1 ! \int_{L} \frac{\varphi(\tau) d \tau}{(\tau-t)^{2}} \\
\ldots & \ldots & \ldots & \ldots & \ldots \\
p_{1}^{(m)}(t) & p_{2}^{(m)}(t) & \ldots & p_{m}^{(m)}(t) & m ! \int_{L} \frac{\varphi(\tau) d \tau}{(\tau-t)^{m+1}}
\end{array}\right|- \\
& -\frac{b(t)}{\pi i}\left|\begin{array}{ccccc}
q_{1}(t) & q_{2}(t) & \ldots & q_{n}(t) & 0 ! \int_{L} \frac{\varphi(\tau) d \tau}{\tau-t} \\
q_{1}^{\prime}(t) & q_{2}^{\prime}(t) & \ldots & q_{n}^{\prime}(t) & 1 ! \int_{L} \frac{\varphi(\tau) d \tau}{(\tau-t)^{2}} \\
\ldots & \ldots & \ldots & \ldots & \ldots \\
q_{1}^{(n)}(t) & q_{2}^{(n)}(t) & \ldots & q_{n}^{(n)}(t) & n ! \int_{L} \frac{\varphi(\tau) d \tau}{(\tau-t)^{n+1}}
\end{array}\right|=f(t), t \in L,
\end{aligned}
$$

с интегралами, понимаемыми в смысле конечной части по Адамару.

Будем обозначать через $W$ вронскиан функций, указывая в скобках сами функции и их аргумент. Для $t \in L$ введем обозначения

$$
\begin{gathered}
W_{+}(t)=W\left(p_{1}, p_{2}, \ldots, p_{m} ; t\right), W_{j}^{+}(t)=W\left(p_{1}, \ldots, p_{j-1}, p_{j+1}, \ldots, p_{m} ; t\right), j=\overline{1, m}, \\
W_{-}(t)=W\left(q_{1}, q_{2}, \ldots, q_{n} ; t\right), W_{j}^{-}(t)=W\left(q_{1}, \ldots, q_{j-1}, q_{j+1}, \ldots, q_{n} ; t\right), j=\overline{1, n} .
\end{gathered}
$$


Пусть точка $z_{0}$ является для некоторой функции точкой аналитичности либо полюсом. Напомним, что порядком этой функции в точке $z_{0}$ называется число $v \in \mathbb{Z}$ из разложения функции в ряд $\sum_{k=v}^{\infty} c_{k}\left(z-z_{0}\right)^{k}$, $c_{v} \neq 0$, в окрестности этой точки.

Возможность решить уравнение (1) анонсирована в статье [7]. В настоящей работе приведем подробно соответствующий результат, допуская к тому же наличие у исходных функций особых точек, значительно усложняющих решение. Как и в статье [7], в дальнейшем будем считать, что все функции $p_{j}(t)$ (а следовательно, и $\left.W_{+}(t), W_{j}^{+}(t)\right)$ аналитически продолжимы в $D_{+}$, причем $W_{+}(z) \neq 0, z \in D_{+} \cup L$. Но сделаем исключение для одной (особой) точки $z_{*} \in D_{+}$: пусть в этой точке порядки функций $p_{j}(z)$ равны $k_{j} \in \mathbb{Z}$ (и тогда необязательно $\left.W_{+}\left(z_{*}\right) \neq 0\right)$. Как и в работе [7], будем предполагать также, что все функции $q_{j}(t)$ (а значит, и $\left.W_{-}(t), W_{j}^{-}(t)\right)$ аналитически продолжимы в $D_{-}$, причем $W_{-}(z) \neq 0, z \in\left(D_{-} \cup L\right) \backslash\{\infty\}$. Роль особой точки в $D_{-}$будет играть точка $z=\infty$, поскольку всегда $W_{-}(\infty)=0$. В статье [7] изучен случай, когда $\Delta \neq 0$, где

$$
\Delta=\left|\begin{array}{cccc}
k_{10} & k_{20} & \ldots & k_{n 0} \\
k_{11} & k_{21} & \ldots & k_{n 1} \\
\ldots & \ldots & \ldots & \ldots \\
k_{1, n-1} & k_{2, n-1} & \ldots & k_{n, n-1}
\end{array}\right|
$$

а элементы определителя $\Delta$ берутся из разложений в ряды Тейлора $q_{j}(z)=\sum_{s=0}^{\infty} \frac{k_{j s}}{z^{s}}$ функций $q_{j}(z)$ в окрестности точки $z=\infty$. Теперь предположим, что функции $q_{j}(z)$ имеют в точке $z=\infty$ нули некоторых порядков $l_{j} \in \mathbb{N}, j=\overline{1, n}$ (и тогда всегда будет $\Delta=0$ ).

\section{Основной результат}

Лемма 1. Если порядки $k_{j}$ функций $p_{j}(z), j=\overline{1, m}$, в точке $z_{*}$ попарно различньл, то порядок $W_{+}(z)$ в этой точке равен $\sum_{j=1}^{m} k_{j}-\frac{(m-1) m}{2}$.

Д о каз ат е л ь т в о. Разложим в точке $z_{*}$ элементы определителя $W_{+}(z)$ в ряды Лорана (в частности, это могут быть ряды Тейлора):

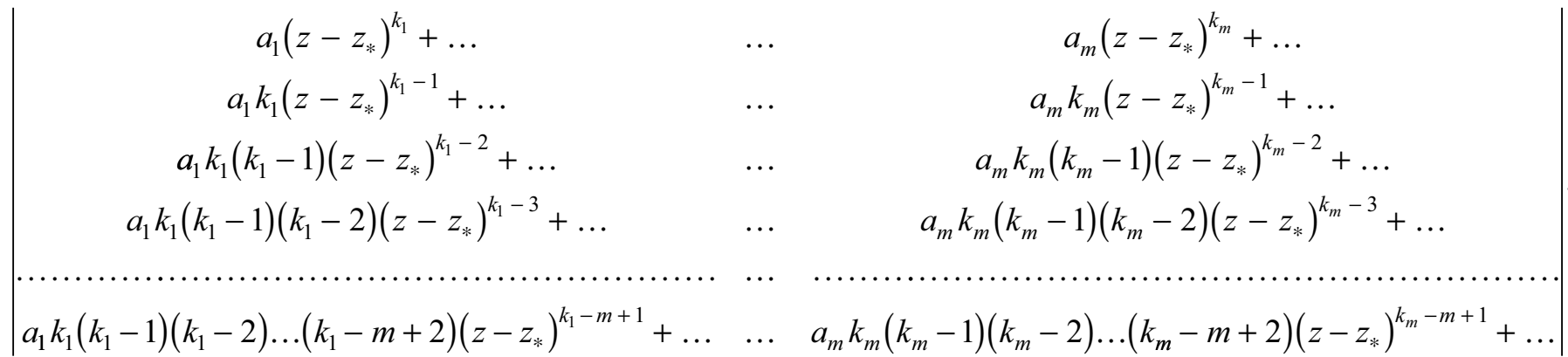

В каждом элементе определителя указаны лишь начальные слагаемые соответствующего ряда, остальные слагаемые обозначены многоточиями, $a_{1}, \ldots, a_{m}$ - ненулевые постоянные. Подчеркнем, что при дифференцировании членов рядов мы пользуемся формулой вида $\left(\left(z-z_{*}\right)^{s}\right)^{(k)}=s(s-1) \ldots(s-k+$ $+1)\left(z-z_{*}\right)^{s-k}$ в том числе и при $0 \leq s<k$, когда $\left(\left(z-z_{*}\right)^{s}\right)^{(k)}=0$. Вынесем за знак определителя множитель $a_{j}\left(z-z_{*}\right)^{k_{j}-m+1}$ из $j$-го столбца, затем множитель $\left(z-z_{*}\right)^{m-j}$ из $j$-й строки, $j=\overline{1, m}$. В резуль-

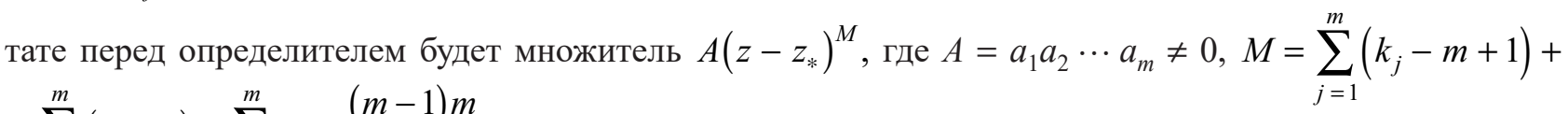
$+\sum_{j=1}^{m}(m-j)=\sum_{j=1}^{m} k_{j}-\frac{(m-1) m}{2}$, а определитель после раскрытия скобок и приведения подобных членов примет вид 


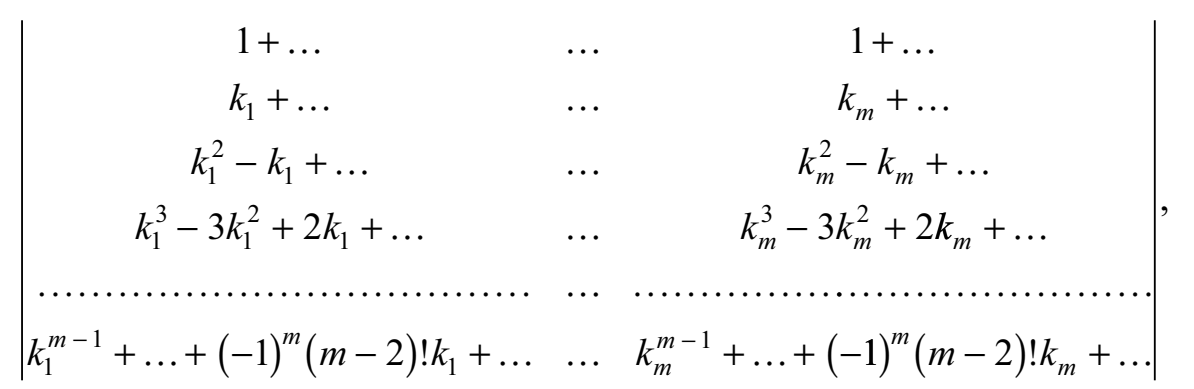

где все многоточия в элементах определителя обозначают бесконечно малые функции при $z \rightarrow z_{*}$.

Теперь полученный определитель представим в виде надлежащей суммы таких определителей, элементами которых будут отдельные слагаемые строк. Ненулевым будет лишь определитель из всех первых слагаемых строк, поскольку он совпадает с определителем $V$ Вандермонда попарно различных чисел $k_{1}, k_{2}, \ldots, k_{m}$. Остальные определители или обратятся в ноль из-за пропорциональности какихлибо строк, или будут бесконечно малыми при $z \rightarrow z_{*}$ из-за наличия таких бесконечно малых хотя бы в одной строке.

Итак,

Лемма доказана.

$$
W_{+}(z)=A V\left(z-z_{*}\right)^{M}+o\left(\left(z-z_{*}\right)^{M}\right), z \rightarrow z_{*} .
$$

Отметим, что при выполнении условий леммы порядки $W_{j}^{+}(z)$ в точке $z_{*}$, очевидно, будут равны $\sum_{\substack{s=1 \\ s \neq j}}^{m} k_{s}-\frac{(m-2)(m-1)}{2}, j=\overline{1, m}$.

Проанализируем для определителя $W_{+}(z)$ случай произвольных целых порядков $k_{j}, j=\overline{1, m}$. Обозначим $\tilde{k}_{1}=k_{j_{1}}$ наименьший из этих порядков. Если наряду с функцией $p_{j_{1}}(z)$ некоторые из функций $p_{j}(z)$ также имеют этот порядок, то прибавим к столбцам определителя $W_{+}(z)$, содержащим такие функции, столбец с функцией $p_{j_{1}}(z)$, умноженный на надлежащие константы, так, чтобы в результате столбец с номером $j_{1}$ остался единственным, в котором порядок первой функции равен $\tilde{k}_{1}$. Обозначим $\tilde{p}_{j}(z)$ функции в первой строке нового определителя, $j=\overline{1, m}$. В частности, будет $\tilde{p}_{j_{1}}(z) \equiv p_{j_{1}}(z)$. Если функция $p_{j_{1}}(z)$ окажется единственной с порядком $\tilde{k}_{1}$ в точке $z_{*}$, то просто считаем $\tilde{p}_{j}(z) \equiv p_{j}(z), j=\overline{1, m}$.

Теперь в новом определителе обозначим $\tilde{k}_{2}$ наименьший порядок в точке $z_{*}$ всех функций $\tilde{p}_{j}(z)$, кроме функции $\tilde{p}_{j_{1}}(z)$, и пусть функция $\tilde{p}_{j_{2}}(z)$ имеет этот порядок. Если некоторые из функций $\tilde{p}_{j}(z)$ также имеют этот порядок в точке $z_{*}$, то прибавим к столбцам с такими функциями столбец с функцией $\tilde{p}_{j_{2}}(z)$, умноженный на надлежащие константы, так, чтобы в результате столбец с номером $j_{2}$ остался единственным, в котором порядок первой функции равен $\tilde{k}_{2}$. Обозначим $\tilde{\tilde{p}}_{j}(z)$ функции в первой строке нового определителя. Если функция $\tilde{p}_{j_{2}}(z)$ будет единственной с порядком $\tilde{k}_{2}$ в точке $z_{*}$, то просто считаем $\tilde{\tilde{p}}_{j}(z) \equiv \tilde{p}_{j}(z)$ для всех $j=\overline{1, m}$. Обозначим $\tilde{k}_{3}$ наименьший порядок в точке $z_{*}$ всех функций $\tilde{\tilde{p}}_{j}(z)$, кроме функций $\tilde{\tilde{p}}_{j_{1}}(z)$ и $\tilde{\tilde{p}}_{j_{2}}(z)$, и продолжим действия со столбцами, аналогичные предыдущим. В результате мы добьемся того, что порядки в точке $z_{*}$ всех функций в первой строке определителя станут попарно различными. Поскольку значение определителя в результате указанных действий со столбцами не изменится, то можно, не теряя общности, считать с самого начала все порядки $k_{j}$ попарно различными, что мы и будем предполагать в дальнейших рассуждениях.

Лемма 2. Если порядки $l_{j}$ нуля на бесконечности функций $q_{j}(z), j=\overline{1, n}$, попарно различны, то порядок нуля на бесконечности $W_{-}(z)$ равен $\sum_{j=1}^{n} l_{j}+\frac{(n-1) n}{2}$.

Подробное доказательство не приводится, поскольку оно аналогично доказательству леммы 1. Отметим только, что, прежде чем проводить похожие рассуждения, элементы первой строки определителя $W_{-}(z)$ следует представить в виде $b_{j} z^{-l_{j}}+\ldots, b_{j} \in \mathbb{C}, b_{j} \neq 0, j=\overline{1, n}$. Получим, что $W_{-}(z)$ относительно $z$ будет иметь степень на бесконечности $\sum_{j=1}^{n}\left(-l_{j}\right)-\frac{(n-1) n}{2}$, т. е. точка $z=\infty$ будет нулем порядка 
$\sum_{j=1}^{n} l_{j}+\frac{(n-1) n}{2}$. Укажем также, что для определителей $W_{j}^{-}(z)$ нуль на бесконечности будет иметь порядки $\sum_{\substack{s=1 \\ s \neq j}}^{n} l_{s}+\frac{(n-2)(n-1)}{2}, j=\overline{1, n}$. Так же как и в случае определителя $W_{+}(z)$, в дальнейшем можно, не теряя общности, всегда считать для определителя $W_{-}(z)$ все порядки $l_{j}$ попарно различными.

Введем новые неизвестные функции:

$$
\begin{gathered}
\Phi_{ \pm}(z)=\frac{1}{2 \pi i} \int_{L} \frac{\varphi(\tau) d \tau}{\tau-z}, z \in D_{ \pm}, \\
F_{+}(z)=W\left(p_{1}, p_{2}, \ldots, p_{m}, \Phi_{+} ; z\right), z \in D_{+}, \\
F_{-}(z)=W\left(q_{1}, q_{2}, \ldots, q_{n}, \Phi_{-} ; z\right), z \in D_{-} .
\end{gathered}
$$

В определителе $W\left(p_{1}, p_{2}, \ldots, p_{m}, \Phi_{+} ; z\right)$ без потери общности рассуждений мы можем считать порядки функций $p_{j}(z)$ в точке $z_{*}$ попарно различными и равными $k_{j}, j=\overline{1, m}$. В противном случае к этому можно прийти теми же действиями с первыми $m$ столбцами, что и в определителе $W_{+}(z)$ : данные действия не изменят определитель точно так же, как и определитель $W_{+}(z)$. Аналогичное замечание справедливо для определителя в уравнении (3).

Функции $\Phi_{ \pm}(z)$ аналитичны в соответствующих областях $D_{ \pm}, \Phi_{-}(\infty)=0$. Функция $F_{+}(z)$ аналитична в $D_{+}$, кроме, возможно, точки $z=z_{*}$, где у нее может быть полюс. Функция $F_{-}(z)$ аналитична в $D_{-}$, $F_{-}(\infty)=0$.

Если порядок функции $\Phi_{+}(z)$ в точке $z_{*}$ не совпадает ни с одним из порядков $k_{j}$ функций $p_{j}(z)$, то порядок $\alpha$ в этой точке у функции $F_{+}(z)$ можно вычислить, применяя лемму 1 к функциям $p_{1}(z), p_{2}(z), \ldots, p_{m}(z), \Phi_{+}(z)$. Для определенности в дальнейшем будем полагать $k_{j} \neq 0, j=\overline{1, m}$. При этом минимально возможный порядок $\alpha=\sum_{j=1}^{m} k_{j}-\frac{m(m+1)}{2}$ будет в случае, когда у функции $\Phi_{+}(z)$ нулевой порядок. Если порядок функции $\Phi_{+}(z)$ в точке $z_{*}$ совпадает с одним из порядков $k_{j}$, то для расчета порядка в этой точке у функции $F_{+}(z)$ используем описанную ранее процедуру действий со столбцами $F_{+}(z)$. При этом допускается изменять лишь последний столбец, а другие оставлять неизменными, из-за чего порядок первого элемента в последнем столбце будет увеличиваться. Функция $F_{+}(z)$ при этом не изменится, а ее порядок в точке $z_{*}$ окажется больше $\alpha$. Таким образом, $F_{+}(z)=O\left(\left(z-z_{*}\right)^{\alpha}\right), z \rightarrow z_{*}$. Аналогично, полагая в дальнейшем $l_{j} \neq 1, j=\overline{1, n}$, с помощью леммы 2 получим $F_{-}(z)=O\left(\left(\frac{1}{z}\right)^{\beta}\right), z \rightarrow \infty$, где $\beta=\sum_{j=1}^{n} l_{j}+\frac{n(n+1)}{2}+1$ (единица, в сравнении с числом $\alpha$, добавится из-за минимально возможного 1-го порядка $F_{-}(z)$ на бесконечности). Используя терминологию [8], можно сказать, что кусочно-аналитические функции $F_{+}(z), F_{-}(z)$ должны быть кратны дивизору $z_{*}^{\alpha} \infty^{\beta}$.

Вернемся к уравнению (1). Его можно сначала записать в виде

$$
a(t)\left|\begin{array}{ccccc}
p_{1}(t) & p_{2}(t) & \ldots & p_{m}(t) & \frac{1}{2} \varphi(t)+\frac{0 !}{2 \pi i} \int_{L} \frac{\varphi(\tau) d \tau}{\tau-t} \\
p_{1}^{\prime}(t) & p_{2}^{\prime}(t) & \ldots & p_{m}^{\prime}(t) & \frac{1}{2} \varphi^{\prime}(t)+\frac{1 !}{2 \pi i} \int_{L} \frac{\varphi(\tau) d \tau}{(\tau-t)^{2}} \\
\ldots & \ldots & \ldots & \ldots & \ldots \\
p_{1}^{(m)}(t) & p_{2}^{(m)}(t) & \ldots & p_{m}^{(m)}(t) & \frac{1}{2} \varphi^{(m)}(t)+\frac{m !}{2 \pi i} \int_{L} \frac{\varphi(\tau) d \tau}{(\tau-t)^{m+1}}
\end{array}\right|=
$$




$$
=b(t)\left|\begin{array}{ccccc}
q_{1}(t) & q_{2}(t) & \ldots & q_{n}(t) & -\frac{1}{2} \varphi(t)+\frac{0 !}{2 \pi i} \int_{L} \frac{\varphi(\tau) d \tau}{\tau-t} \\
q_{1}^{\prime}(t) & q_{2}^{\prime}(t) & \ldots & q_{n}^{\prime}(t) & -\frac{1}{2} \varphi^{\prime}(t)+\frac{1 !}{2 \pi i} \int_{L} \frac{\varphi(\tau) d \tau}{(\tau-t)^{2}} \\
\ldots & \ldots & \ldots & \ldots & \ldots \\
q_{1}^{(n)}(t) & q_{2}^{(n)}(t) & \ldots & q_{n}^{(n)}(t) & -\frac{1}{2} \varphi^{(n)}(t)+\frac{n !}{2 \pi i} \int_{L} \frac{\varphi(\tau) d \tau}{(\tau-t)^{n+1}}
\end{array}\right|+\frac{f(t)}{2}, t \in L,
$$

а потом, используя обобщенные формулы Сохоцкого [9], в виде краевой задачи Римана

$$
F_{+}(t)=\frac{b(t)}{a(t)} F_{-}(t)+\frac{f(t)}{2 a(t)}, t \in L .
$$

Если решение этой задачи будет найдено, то соотношения (2), (3) следует затем расценивать как линейные дифференциальные уравнения для нахождения функций $\Phi_{ \pm}(z)$. В случае если и функции $\Phi_{ \pm}(z)$ будут найдены, решение уравнения (1) можно найти по формуле

$$
\varphi(t)=\Phi_{+}(t)-\Phi_{-}(t), t \in L .
$$

Таким образом, справедлива следующая теорема.

Теорема. Решение уравнения (1) сводится к последовательному решению краевой задачи Римана (4) в классе функиий, кратных дивизору $z_{*}^{\alpha} \infty^{\beta}$, и дифференциальных уравнений (2), (3) в классе аналитических функиий (с условием $\left.\Phi_{-}(\infty)=0\right)$.

\section{Дальнейшее исследование}

Решение задачи Римана. Теория задачи Римана [10] позволяет решить возникшую задачу (4). Введем следующие обозначения: $\varkappa=\operatorname{Ind}_{L} \frac{b(t)}{a(t)} ; X_{ \pm}(z)$ - канонические функции задачи Римана, факторизующие ее коэффициент $\frac{b(t)}{a(t)} ; \Psi_{ \pm}(z)=\frac{1}{4 \pi i} \int_{L} \frac{f(\tau) d \tau}{a(\tau) X_{+}(\tau)(\tau-z)}, z \in D_{ \pm}$. Решение задачи (4) имеет вид

$$
F_{ \pm}(z)=X_{ \pm}(z)\left(P_{\varkappa-\beta}(z)+\frac{\Psi_{ \pm}(z)\left(z-z_{*}\right)^{|\alpha|}+Q_{|\alpha|-1}(z)}{\left(z-z_{*}\right)^{|\alpha|}}\right), z \in D_{ \pm} \text {. }
$$
При $\varkappa-\beta<0 P_{\varkappa-\beta}(z) \equiv 0$, а при $\varkappa-\beta \geq 0 P_{\varkappa-\beta}(z)-$ многочлен вида $\sum_{k=0}^{\varkappa-\beta} c_{k}\left(z-z_{*}\right)^{k}$, коэффициенты
которого зависят от величины $\alpha$ :

- в случае $\alpha \leq 0$ являются произвольными;

- в случае $\alpha>\varkappa-\beta$ вычисляются по формулам

$$
c_{k}=\frac{(-1)^{k+1}}{4 \pi i} \int_{L} \frac{f(\tau) d \tau}{a(\tau)\left(\tau-z_{*}\right)^{k+1}}
$$

для всех $k=\overline{0, \varkappa-\beta}$;

• в случае $1 \leq \alpha \leq \varkappa-\beta$ вычисляются по формулам (6) для $k=\overline{0, \alpha-1}$, а для $k=\overline{\alpha, \varkappa-\beta}$ остаются произвольными. Если $\alpha \geq 0$, то $Q_{|\alpha|-1}(z) \equiv 0$, если же $\alpha \leq-1$, то $Q_{|\alpha|-1}(z)$ - многочлен вида $\sum_{k=0}^{|\alpha|-1} d_{k}\left(z-z_{*}\right)^{k}$, коэффи-
циенты которого зависят от величины $\varkappa-\beta$ :

- в случае $\varkappa-\beta \geq-1$ являются произвольными;

• в случае $\varkappa-\beta \leq-|\alpha|-1$ вычисляются по формулам 
для всех $k=\overline{1,|\alpha|}$;

$$
d_{|\alpha|-k}=\frac{1}{4 \pi i} \int_{L} \frac{f(\tau)\left(\tau-z_{*}\right)^{k-1} d \tau}{a(\tau)}
$$

- в случае $-|\alpha| \leq \varkappa-\beta \leq-2$ вычисляются по формулам (7) для $k=\overline{1, \beta-\varkappa-1}$, а для $k=\overline{\beta-\varkappa,|\alpha|}$ остаются произвольными.

При этом для разрешимости задачи (4) необходимым и достаточным является:

1) при $1 \leq \varkappa-\beta+1<\alpha$ выполнение условий

в которых $k=\overline{\varkappa-\beta+1, \alpha-1}$;

$$
\int_{L} \frac{f(\tau) d \tau}{a(\tau) X_{+}(\tau)\left(\tau-z_{*}\right)^{k+1}}=0
$$

2) при $\varkappa-\beta<-|\alpha|-1, \alpha \leq-1$ выполнение условий

$$
\int_{L} \frac{f(\tau)\left(\tau-z_{*}\right)^{k-1} d \tau}{a(\tau)}=0
$$

в которых $k=\overline{|\alpha|+1, \beta-\varkappa-1}$;

3) при $\varkappa-\beta<-1, \alpha>0$ выполнение совокупности условий (8), в которых $k=\overline{0, \alpha-1}$, и условий (9), в которых $k=\overline{1, \beta-\varkappa-1}$.

В остальных случаях, т. е. при $\varkappa-\beta-\alpha \geq-1$, задача разрешима безусловно.

Решение дифференциальных уравнений. Предполагая, что задача Римана (4) разрешима, а ее решение найдено, будем исследовать в области $D_{+}$уравнение (2). Решение этого уравнения, полученное методом вариации произвольных постоянных, имеет вид

$$
\Phi_{+}(z)=\sum_{j=1}^{m}\left(C_{j}^{+} p_{j}(z)+p_{j}(z) \tilde{C}_{j}(z)\right)
$$

где $C_{j}^{+} \in \mathbb{C}, \tilde{C}_{j}(z)$ - какие-либо первообразные функций $\frac{W_{j}^{+}(z) F_{+}(z)}{W_{+}^{2}(z)}, j=\overline{1, m}$. Для дальнейших рассуждений зафиксируем точку $z_{0}^{+} \in D_{+}, z_{0}^{+} \neq z_{*}$. Для определенности будем считать, что для $j=\overline{1, \gamma}$ $k_{j} \geq 0$, а для $j=\overline{\gamma+1, m} k_{j}<0$. На основании порядков в точке $z_{*}$ функций $W_{j}^{+}(z), F_{+}(z), W_{+}(z)$, установленных в лемме 1 , и последующих после ее доказательства рассуждений заключаем, что порядок функции $\frac{W_{j}^{+}(z) F_{+}(z)}{W_{+}^{2}(z)}$ не ниже

$$
\sum_{\substack{s=1 \\ s \neq j}}^{m} k_{s}-\frac{(m-2)(m-1)}{2}+\sum_{j=1}^{m} k_{j}-\frac{(m-1) m}{2}-2\left(\sum_{j=1}^{m} k_{j}-\frac{m(m+1)}{2}\right)=-k_{j}-1 .
$$

Теперь понятно, что формула (10), вообще говоря, не даст аналитическую функцию в $D_{+}$по трем причинам.

Во-первых, для $j=\overline{1, \gamma}$ упомянутые производные могут не существовать. Для их существования необходимо и достаточно выполнения условий

$$
\operatorname{res}_{z=z_{*}} \frac{W_{j}^{+}(z) F_{+}(z)}{W_{+}^{2}(z)}=0, j=\overline{1, \gamma} .
$$

Если эти условия выполняются, то в качестве нужных первообразных можно брать функции $\tilde{C}_{j}(z)=\int_{z_{0}^{+}}^{z} \frac{W_{j}^{+}(\zeta) F_{+}(\zeta) d \zeta}{W_{+}^{2}(\zeta)}$, в которых интегралы вычисляются по любым кривым в $D_{+}$, не проходящим через точку $z_{*}$. Полюсы порядков не более $k_{j}$ для $k_{j}>0$ у этих первообразных «погасятся» в формуле (10) нулями порядков $k_{j}$ соответствующих множителей $p_{j}(z)$.

Во-вторых, слагаемые $C_{j}^{+} p_{j}(z)$ для $j=\overline{\gamma+1, m}$ в формуле (10) дадут полюс в точке $z_{*}$. Чтобы его устранить, для этих $j$ следует брать $C_{j}^{+}=0$.

$$
\text { 52Y - cmovemorar ucmopura yonexa }
$$


В-третьих, слагаемые $p_{j}(z) \tilde{C}_{j}(z)$ для $j=\overline{\gamma+1, m}$ в формуле (10) также могут дать полюс в точке $z_{*}$ из-за множителей $p_{j}(z)$. Взяв для этих $j$ первообразные в виде $\int_{z_{*}}^{z} \frac{W_{j}^{+}(\zeta) F_{+}(\zeta) d \zeta}{W_{+}^{2}(\zeta)}$, мы получим у данных интегралов нули порядков не ниже $-k_{j}$ в точке $z_{*}$, «гасящие» полюсы.

Итак, для решения дифференциального уравнения (2) в классе аналитических в $D_{+}$функций необходимо и достаточно выполнения условий (11). При их выполнении решение записывается по формуле

$$
\Phi_{+}(z)=\sum_{j=1}^{\gamma} p_{j}(z)\left(C_{j}^{+}+\int_{z_{0}^{+}}^{z} \frac{W_{j}^{+}(\zeta) F_{+}(\zeta) d \zeta}{W_{+}^{2}(\zeta)}\right)+\sum_{j=\gamma+1}^{m} p_{j}(z) \int_{z_{*}}^{z} \frac{W_{j}^{+}(\zeta) F_{+}(\zeta) d \zeta}{W_{+}^{2}(\zeta)} .
$$

Рассуждения при решении уравнения (3) аналогичны. Для существования этого решения (с учетом условия $\left.\Phi_{-}(\infty)=0\right)$ необходимо и достаточно выполнения условий

$$
\operatorname{res}_{z=\infty} \frac{W_{j}^{-}(z) F_{-}(z)}{W_{-}^{2}(z)}=0, j=\overline{1, n} .
$$

При их выполнении решение записывается по формуле

$$
\Phi_{-}(z)=\sum_{j=1}^{n} q_{j}(z)\left(C_{j}^{-}+\int_{z_{0}^{-}}^{z} \frac{W_{j}^{-}(\zeta) F_{-}(\zeta) d \zeta}{W_{-}^{2}(\zeta)}\right),
$$

где $C_{j}^{-}$- произвольные постоянные, $z_{0}^{-}$- фиксированная точка, $z_{0}^{-} \in D_{-}, z_{0}^{-} \neq \infty$, а интегралы берутся по любым кривым в $D_{-}$, не проходящим через точку $z=\infty$.

Теперь при разных значениях $\varkappa, \alpha, \beta$ теореме можно придавать развернутые формулировки, которые необходимо расценивать как следствия из теоремы. В формулировках надо учитывать, что равенства (11), (12) при наличии произвольных постоянных в формулах для $F_{ \pm}(z)$ станут линейными алгебраическими уравнениями. Например, будет справедливо нижеприведенное следствие.

Следствие. При $\varkappa-\beta \geq 0, \alpha \leq-1$ для разрешимости уравнения (1) необходима и достаточна совместность системь

$$
\left\{\begin{array}{l}
\sum_{k=0}^{\varkappa-\beta} \alpha_{j k} c_{k}+\sum_{k=0}^{|\alpha|-1} \beta_{j k} d_{k}=A_{j}, j=\overline{1, \gamma} \\
\varkappa-\beta \\
\sum_{k=0} \gamma_{j k} c_{k}+\sum_{k=0}^{|\alpha|-1} \delta_{j k} d_{k}=B_{j}, j=\overline{1, n}
\end{array}\right.
$$

где для всех указанных $j, k$

$$
\begin{gathered}
\alpha_{j k}=\operatorname{res}_{z=z_{*}} \frac{W_{j}^{+}(z) X_{+}(z)\left(z-z_{*}\right)^{k}}{W_{+}^{2}(z)}, \beta_{j k}=\underset{z=z_{*}}{\operatorname{res}} \frac{W_{j}^{+}(z) X_{+}(z)}{W_{+}^{2}(z)\left(z-z_{*}\right)^{|\alpha|-k}}, \\
A_{j}=-\operatorname{res}_{z=z_{*}} \frac{W_{j}^{+}(z) X_{+}(z) \Psi_{+}(z)}{W_{+}^{2}(z)}, \gamma_{j k}=\operatorname{res}_{z=\infty} \frac{W_{j}^{-}(z) X_{-}(z)\left(z-z_{*}\right)^{k}}{W_{-}^{2}(z)}, \\
\delta_{j k}=\operatorname{res}_{z=\infty} \frac{W_{j}^{-}(z) X_{-}(z)}{W_{-}^{2}(z)\left(z-z_{*}\right)^{|\alpha|-k}}, \quad B_{j}=-\operatorname{res}_{z=\infty} \frac{W_{j}^{-}(z) X_{-}(z) \Psi_{-}(z)}{W_{-}^{2}(z)} .
\end{gathered}
$$

В случае совместности системы (13) решение уравнения записывается по формуле

$$
\begin{aligned}
\varphi(t)=\sum_{j=1}^{\gamma} p_{j}(t)\left(C_{j}^{+}\right. & \left.+\int_{z_{0}^{+}}^{t} \frac{W_{j}^{+}(\zeta) F_{+}(\zeta) d \zeta}{W_{+}^{2}(\zeta)}\right)+\sum_{j=\gamma+1}^{m} p_{j}(t) \int_{z_{*}}^{t} \frac{W_{j}^{+}(\zeta) F_{+}(\zeta) d \zeta}{W_{+}^{2}(\zeta)}- \\
& -\sum_{j=1}^{n} q_{j}(t)\left(C_{j}^{-}+\int_{z_{0}^{-}}^{t} \frac{W_{j}^{-}(\zeta) F_{-}(\zeta) d \zeta}{W_{-}^{2}(\zeta)}\right)
\end{aligned}
$$


в которой постоянные $c_{k}, d_{k}$, входящие в выражения для $F_{ \pm}(t)$, являются общии решением системы (13).

Аналогичные утверждения для иных $\varkappa, \alpha, \beta$ приводить не будем.

Пример. Решим уравнение

$$
\begin{aligned}
& \left|\begin{array}{ccc}
t & \sin t & \varphi(t) \\
1 & \cos t & \varphi^{\prime}(t) \\
0 & -\sin t & \varphi^{\prime \prime}(t)
\end{array}\right|+(t+1)^{10}\left|\begin{array}{ccc}
\frac{1}{t^{2}} & \frac{2 t-1}{2 t^{4}} & \varphi(t) \\
-\frac{2}{t^{3}} & \frac{2-3 t}{t^{5}} & \varphi^{\prime}(t) \\
\frac{6}{t^{4}} & \frac{2(6 t-5)}{t^{6}} & \varphi^{\prime \prime}(t)
\end{array}\right|+
\end{aligned}
$$

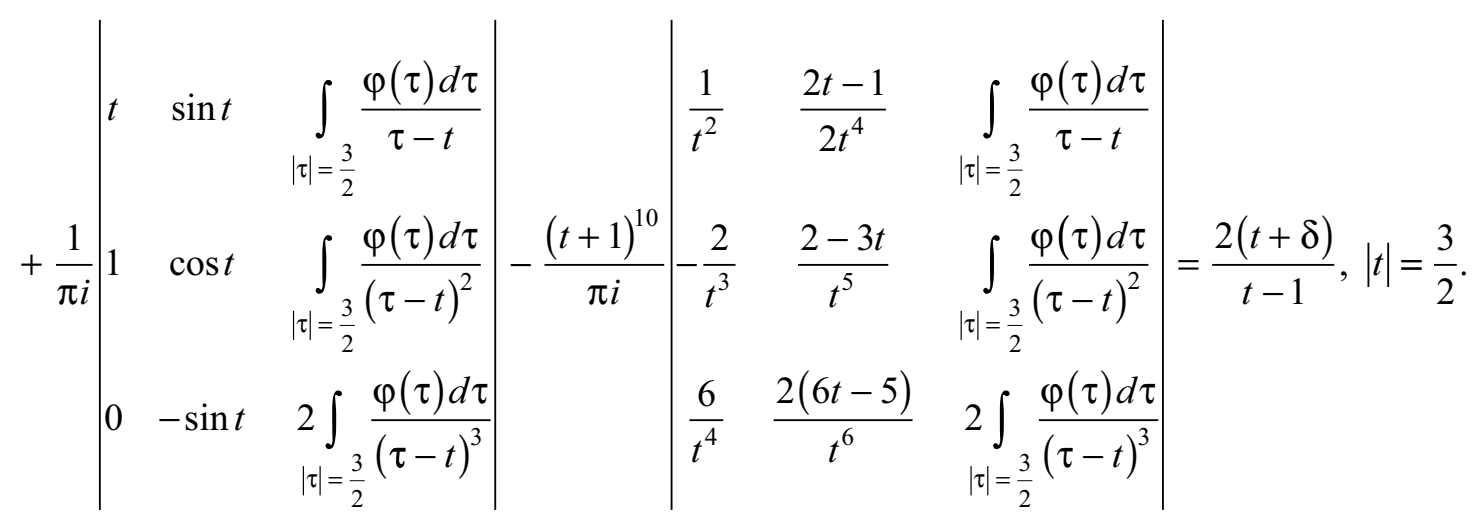

Здесь $m=n=2, \delta$ - числовой параметр, $W_{+}(z)=z \cos z-\sin z, W_{-}(z)=\frac{1-z}{z^{7}}$. Точка $z=\infty$, очевидно, будет единственной особой точкой в области $D_{-}=\left\{z|| z \mid>\frac{3}{2}\right\}$. В области $D_{+}=\left\{z|| z \mid<\frac{3}{2}\right\}$ роль особой точки играет точка $z_{*}=0$, однако единственность такой точки нуждается в обосновании.

Рассмотрим функцию $z \cos z-\sin z$ внутри квадрата с центром в нуле, стороны которого имеют длину $\frac{3 \pi}{2}$ и параллельны действительной и мнимой осям. Несложно вычислить, что для всех точек на сторонах квадрата справедливо неравенство $|z \cos z|>|\sin z|$. По теореме Руше получается, что в этом квадрате число нулей функции $z \cos z$ равно числу нулей функции $z \cos z-\sin z$. Функция $z \cos z$, очевидно, имеет нули лишь в точках $0, \pm \frac{\pi}{2}$ квадрата, а в область $D_{+}$попадает только точка $z_{*}=0$. (В связи с нулями функции $z \cos z-\sin z$ см. также пример 23.17 в сборнике задач [11].)

Краевое условие задачи Римана (4) примет вид

$$
\left|\begin{array}{ccc}
t-\sin t & \sin t & \Phi_{+}(t) \\
1-\cos t & \cos t & \Phi_{+}^{\prime}(t) \\
\sin t & -\sin t & \Phi_{+}^{\prime \prime}(t)
\end{array}\right|=(t+1)^{10}\left|\begin{array}{ccc}
\frac{1}{t^{2}} & \frac{2 t-1}{2 t^{4}} & \Phi_{-}(t) \\
-\frac{2}{t^{3}} & \frac{2-3 t}{t^{5}} & \Phi_{-}^{\prime}(t) \\
\frac{6}{t^{4}} & \frac{2(6 t-5)}{t^{6}} & \Phi_{-}^{\prime \prime}(t)
\end{array}\right|+\frac{t+\delta}{t-1},|t|=\frac{3}{2} .
$$

При этом в определителе, стоящем в левой части (15), исходный первый столбец заменен на разность первого и второго столбцов. Тем самым в согласии с общей схемой созданы разные порядки в точке $z_{*}=0$ у первых элементов этих столбцов. Вычисления показывают, что $m_{1}=3, m_{2}=1, \alpha=1$, $n_{1}=2, n_{2}=3, \beta=9$. Следовательно, краевую задачу Римана

для функций

$$
F_{+}(t)=(t+1)^{10} F_{-}(t)+\frac{t+\delta}{t-1},|t|=\frac{3}{2},
$$




$$
F_{+}(z)=\left|\begin{array}{ccc}
z-\sin z & \sin z & \Phi_{+}(z) \\
1-\cos z & \cos z & \Phi_{+}^{\prime}(z) \\
\sin z & -\sin z & \Phi_{+}^{\prime \prime}(z)
\end{array}\right|, F_{-}(z)=\left|\begin{array}{ccc}
\frac{1}{z^{2}} & \frac{2 z-1}{2 z^{4}} & \Phi_{-}(z) \\
-\frac{2}{z^{3}} & \frac{2-3 z}{z^{5}} & \Phi_{-}^{\prime}(z) \\
\frac{6}{z^{4}} & \frac{2(6 z-5)}{z^{6}} & \Phi_{-}^{\prime \prime}(z)
\end{array}\right|
$$

необходимо решать в классе функций, кратных дивизору $0^{1} \infty^{9}$. В результате получаем

$$
F_{+}(z)=c z, F_{-}(z)=\frac{c z^{2}-(c+1) z-\delta}{(z+1)^{10}(z-1)}, \forall c \in \mathbb{C} .
$$

Теперь следует решать в $D_{+}$дифференциальное уравнение

откуда находим

$$
\left|\begin{array}{ccc}
z-\sin z & \sin z & \Phi_{+}(z) \\
1-\cos z & \cos z & \Phi_{+}^{\prime}(z) \\
\sin z & -\sin z & \Phi_{+}^{\prime \prime}(z)
\end{array}\right|=c z
$$

$$
\begin{gathered}
\Phi_{+}(z)=C_{1}^{+}(z-\sin z)+C_{2}^{+} \sin z+ \\
+c(\sin z-z) \int_{z_{0}^{+}}^{z} \frac{\zeta \sin \zeta d \zeta}{(\zeta \cos \zeta-\sin \zeta)^{2}}+c \sin z \int_{z_{0}^{+}}^{z} \frac{(\zeta-\sin \zeta) \zeta d \zeta}{(\zeta \cos \zeta-\sin \zeta)^{2}} .
\end{gathered}
$$

Обе подынтегральные функции в последней формуле являются четными, поэтому их вычеты в точке $z_{*}=0$ равны нулю, так что интегралы будут давать однозначные функции. Далее следует решать в $D_{-}$ уравнение

$$
\left|\begin{array}{ccc}
\frac{1}{z^{2}} & \frac{2 z-1}{2 z^{4}} & \Phi_{-}(z) \\
-\frac{2}{z^{3}} & \frac{2-3 z}{z^{5}} & \Phi_{-}^{\prime}(z) \\
\frac{6}{z^{4}} & \frac{2(6 z-5)}{z^{6}} & \Phi_{-}^{\prime \prime}(z)
\end{array}\right|=\frac{c z^{2}-(c+1) z-\delta}{(z+1)^{10}(z-1)} .
$$

Решением будет функция

$$
\begin{gathered}
\Phi_{-}(z)=\frac{C_{1}^{-}}{z^{2}}+\frac{C_{2}^{-}(2 z-1)}{2 z^{4}}+\frac{1}{2 z^{2}} \int_{z_{0}^{-}}^{z} \frac{(2 \zeta-1)\left(c \zeta^{2}-(c+1) \zeta-\delta\right) \zeta^{10} d \zeta}{(\zeta+1)^{10}(1-\zeta)^{3}}+ \\
+\frac{2 z-1}{2 z^{4}} \int_{z_{0}^{-}}^{z} \frac{\left(c \zeta^{2}-(c+1) \zeta-\delta\right) \zeta^{12} d \zeta}{(\zeta+1)^{10}(\zeta-1)^{3}},
\end{gathered}
$$

где постоянные $c, \delta$ еще нужно подобрать так, чтобы указанные интегралы давали однозначные функции в $D_{-}$. Для этого необходимо и достаточно выполнения условий

$$
\begin{gathered}
\operatorname{res}_{z=\infty} \frac{(2 z-1)\left(c z^{2}-(c+1) z-\delta\right) z^{10}}{(z+1)^{10}(1-z)^{3}}=-17 c-2=0, \\
\operatorname{res}_{z=\infty} \frac{\left(c z^{2}-(c+1) z-\delta\right) z^{12}}{(z+1)^{10}(z-1)^{3}}=\delta-38 c-7=0,
\end{gathered}
$$

откуда $c=-\frac{2}{17}, \delta=\frac{43}{17}$. Итак, уравнение (14) разрешимо лишь при $\delta=\frac{43}{17}$. Решение при этом значении $\delta$, найденное по формуле (5) с учетом получившегося значения $c$, будет равно 


$$
\begin{gathered}
\varphi(t)=C_{1}^{+}(t-\sin t)+C_{2}^{+} \sin t-\frac{C_{1}^{-}}{t^{2}}-\frac{C_{2}^{-}(2 t-1)}{2 t^{4}}+ \\
+\frac{2}{17}(t-\sin t) \int_{z_{0}^{+}}^{t} \frac{\zeta \sin \zeta d \zeta}{(\zeta \cos \zeta-\sin \zeta)^{2}}-\frac{2 \sin t}{17} \int_{z_{0}^{+}}^{t} \frac{(\zeta-\sin \zeta) \zeta d \zeta}{(\zeta \cos \zeta-\sin \zeta)^{2}}+ \\
+\frac{1}{34 t^{2}} \int_{z_{0}^{-}}^{t} \frac{(2 \zeta-1)\left(2 \zeta^{2}+15 \zeta+43\right) \zeta^{10} d \zeta}{(\zeta+1)^{10}(1-\zeta)^{3}}-\frac{2 t-1}{34 t^{4}} \int_{z_{0}^{-}}^{t} \frac{\left(2 \zeta^{2}+15 \zeta+43\right) \zeta^{12} d \zeta}{(\zeta+1)^{10}(1-\zeta)^{3}},|t|=\frac{3}{2} .
\end{gathered}
$$

Отметим, что два последних интеграла в (16) поддаются дальнейшим вычислениям. Результаты этих вычислений, полученные с помощью подходящих компьютерных программ, приводить в настоящей статье представляется нецелесообразным из-за их громоздкости.

\section{Заключение}

Исследование исходного уравнения носит законченный характер. Дальнейшие разработки возможны для случая нескольких особых точек. По-видимому, можно вводить в рассмотрение и конструктивно исследовать уравнения с определителями, сводящиеся к краевым задачам Гильберта, Карлемана и др.

\section{Библиографические ссылки}

1. Boykov IV, Ventsel ES, Boykova AI. An approximate solution of hypersingular integral equations. Applied Numerical Mathematics. 2010;60(6):607-628. DOI: 10.1016/j.apnum.2010.03.003.

2. Бойков ИВ. Аналитические и численные методы решения гиперсингулярных интегральных уравнений. Динамические системы. 2019;9(3):244-272.

3. Зверович ЭИ. Решение гиперсингулярного интегро-дифференциального уравнения с постоянными коэффициентами. Доклады Национальной академии наук Беларуси. 2010;54(6):5-8.

4. Зверович ЭИ, Шилин АП. Решение интегро-дифференциальных уравнений с сингулярными и гиперсингулярными интегралами специального вида. Известия Наџиональной академии наук Беларуси. Серия физико-математических наук. 2018; 54(4):404-407. DOI: 10.29235/1561-2430-2018-54-4-404-407.

5. Шилин АП. Гиперсингулярное интегро-дифференциальное уравнение эйлерова типа. Известия Национальной академии наук Беларуси. Серия физико-математических наук. 2020;56(1):17-29. DOI: 10.29235/1561-2430-2020-56-1-17-29.

6. Шилин АП. О решении одного интегро-дифференциального уравнения с сингулярным и гиперсингулярным интегралами. Известия Национальной академии наук Беларуси. Серия физико-математических наук. 2020;56(3):298-309. DOI: 10.29235/1561-2430-2020-56-3-298-309.

7. Шилин АП. Дифференциальная краевая задача Римана и ее приложение к интегро-дифференциальным уравнениям. Доклады Национальной академии наук Беларуси. 2019;63(4):391-397. DOI: 10.29235/1561-8323-2019-63-4-391-397.

8. Зверович ЭИ. Краевые задачи теории аналитических функций в гёльдеровских классах на римановых поверхностях. Успехи математических наук. 1971;26(1):113-179.

9. Зверович ЭИ. Обобщение формул Сохоцкого. Известия Национальной академии наук Беларуси. Серия физико-математических наук. 2012;2:24-28.

10. Гахов ФД. Краевые задачи. 3-е издание. Москва: Наука; 1977. 640 с.

11. Евграфов МА, Бежанов КА, Сидоров ЮВ, Федорюк МВ, Шабунин МИ. Сборник задач по теории аналитических функицй. 2-е издание. Евграфов МА, редактор. Москва: Наука; 1972. 416 с.

\section{References}

1. Boykov IV, Ventsel ES, Boykova AI. An approximate solution of hypersingular integral equations. Applied Numerical Mathematics. 2010;60(6):607-628. DOI: 10.1016/j.apnum.2010.03.003.

2. Boykov IV. Analytical and numerical methods for solving hypersingular integral equations. Dinamicheskie sistemy. 2019;9(3): 244-272. Russian.

3. Zverovich EI. Solution of the hypersingular integro-differential equation with constant coefficients. Doklady of the National Academy of Sciences of Belarus. 2010;54(6):5-8. Russian.

4. Zverovich EI, Shilin AP. Integro-differential equations with singular and hypersingular integrals. Proceedings of the National Academy of Sciences of Belarus. Physics and Mathematics Series. 2018;54(4):404-407. Russian. DOI: 10.29235/1561-2430-2018-544-404-407.

5. Shilin AP. A hypersingular integro-differential equation of the Euler type. Proceedings of the National Academy of Sciences of Belarus. Physics and Mathematics Series. 2020;56(1):17-29. Russian. DOI: 10.29235/1561-2430-2020-56-1-17-29.

6. Shilin AP. On the solution of one integro-differential equation with singular and hypersingular integrals. Proceedings of the National Academy of Sciences of Belarus. Physics and Mathematics Series. 2020;56(3):298-309. Russian. DOI: 10.29235/1561-24302020-56-3-298-309. 
7. Shilin AP. Riemann's differential boundary-value problem and its application to integro-differential equations. Doklady of the National Academy of Sciences of Belarus. 2019;63(4):391-397. Russian. DOI: 10.29235/1561-8323-2019-63-4-391-397.

8. Zverovich EI. [Boundary value problem in the theory of analytic functions in Holder classes on Riemann surfaces]. Uspekhi matematicheskikh nauk. 1971;26(1):113-179. Russian.

9. Zverovich EI. Generalization of Sokhotski's formulas. Proceedings of the National Academy of Sciences of Belarus. Physics and Mathematics Series. 2012;2:24-28. Russian.

10. Gakhov FD. Kraevye zadachi [Boundary value problems]. $3^{\text {rd }}$ edition. Moscow: Nauka; 1977. 640 p. Russian.

11. Yevgrafov MA, Bezhanov KA, Sidorov YuV, Fedoryuk MV, Shabunin MI. Sbornik zadach po teorii analiticheskikh funktsii [Collection of problems on the theory of analytic functions]. $2^{\text {nd }}$ edition. Yevgrafov MA, editor. Moscow: Nauka; 1972. 416 p. Russian.

Получена 04.05.2021 / исправлена 02.06.2021 / принята 02.06.2021. Received 04.05.2021 / revised 02.06.2021 / accepted 02.06.2021. 